\title{
Microprogramming in psychology
}

\author{
JACK HOWARD \\ Hewlett-Packard Company, Cupertino, California 95014
}

This paper describes the concept of application microprogramming. The major areas discussed are a definition of microprogramming, the invocation of microprograms from ordinary programming languages, the development cycle of a microprogram from initial design to final implementation, and the data processing tasks suitable for microprogramming. A calculation of "variance" with and without microcode enhancement is used for demonstration.

\section{DEFINITION OF MIICROPROGRAMIMING}

The application-oriented definition of microprogramming is the addition of customized instructions to a CPU so as to achieve reduced execution time and/or special capabilities. Information on the history and more theoretical aspects of microprogramming may be found in Husson (1970).

As an illustration of this definition, and of the power of microprogramming, consider the problem of calculating the variance of data. Ordinarily, this calculation is performed by executing a long series of assembler instructions, some of which are for general arithmetic calculations, none of which are specifically for calculating variance. By designing a customized assembler instruction to calculate variance (i.e., writing a variance calculating microprogram), the CPU is altered from a general purpose computer to one specifically "designed" to calculate variance. This variance programming problem is used as an example throughout the paper. The speed and facilities advantages of customized instructions (i.e., microprograms) over ordinary assembler instructions are due to the following: (1) Reduction of memory fetches required. (2) The per instruction execution time advantage of a microinstruction. (Microinstructions typically execute 5 to 10 times faster than assembler instructions.) (3) The greater number of registers available at the microcode level. (4) The greater number of arithmetic and logical operations available at the microcode level.

It should be noted that microcode execution is performed at the most basic level of computation and therefore precludes all interrupt processing. To avoid losing interrupts, a periodic check must be made (in the microcode) with interrupt servicing at appropriate condition detection.

\section{INVOCATION OF MICROPROGRAMS FROM ORDINARY PROGRAMMING LANGUAGES}

Suppose one were to code a Fortran WRITE statement. The Fortran compiler would generate a series of assembler instructions to perform the WRITE function.
These assembier instructions reside in, and execute out of, main memory. In a microprogrammed CPU, each assembler instruction invokes a series of microinstructions that reside in, and execute out of, control store. This, then, is the hierarchy in a microprogrammed CPU: high-level language to assembler language to microcode.

Figure 1 illustrates this fundamental hardware architectural innovation. In conventional computer design, the control store module is either not present or it is inaccessible. To access a user written microprogram from an assembler language program, a machine language operation code is selected that will "map" to the control store area containing the desired microprogram.

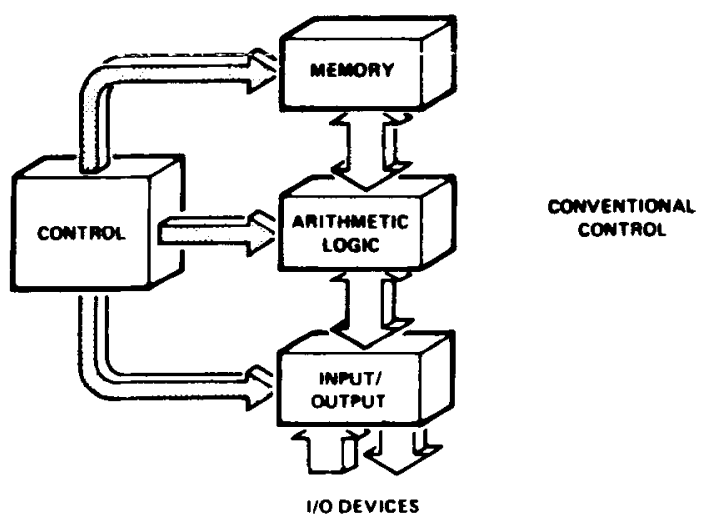

MICROAROGRAMMED CONTROL

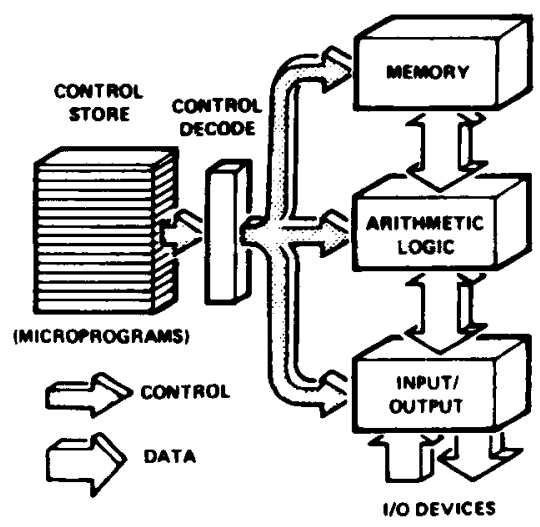

Figure 1, 
To access a user written microprogram from Fortran (i.e., any high-level language), a CALL is coded which results in generation of an assembler jump-to-subroutine instruction. Ordinarily, execution of the CALL invokes a software subroutine that performs some desired function. Instead, a short "swap" routine is substituted for the software subroutine. The swap routine replaces the jump-to-subroutine assembler instruction with the machine language operation code necessary to "map" to the control store area containing the desired microprogram. The swap routine then jumps to this machine language operation code, thereby causing execution of the microprogram (see Figure 2). Note that after the initial swap, the micropiogram executes directly without any further swap routine overhead.

\section{DEVELOPMENT CYCLE OF A MICROPROGRAM FROM INITIAL DESIGN TO INITIAL IMPLEMENTATION}

After selecting the part or parts of programs to be microcoded and writing the microcode, the microprogram is microassembled and loaded into a dynamically modifiable section of control store known as WCS (Writable Control Store). Use of WCS to check out and debug microcode is invariably desirable. It is also desirable to use the facilities of an editor specifically designed to load WCS and monitor microcode execution. For example, Hewlett-Packard has a standard piece of software known as MDC (Micro Debuy Editoi) which serves this purpose.

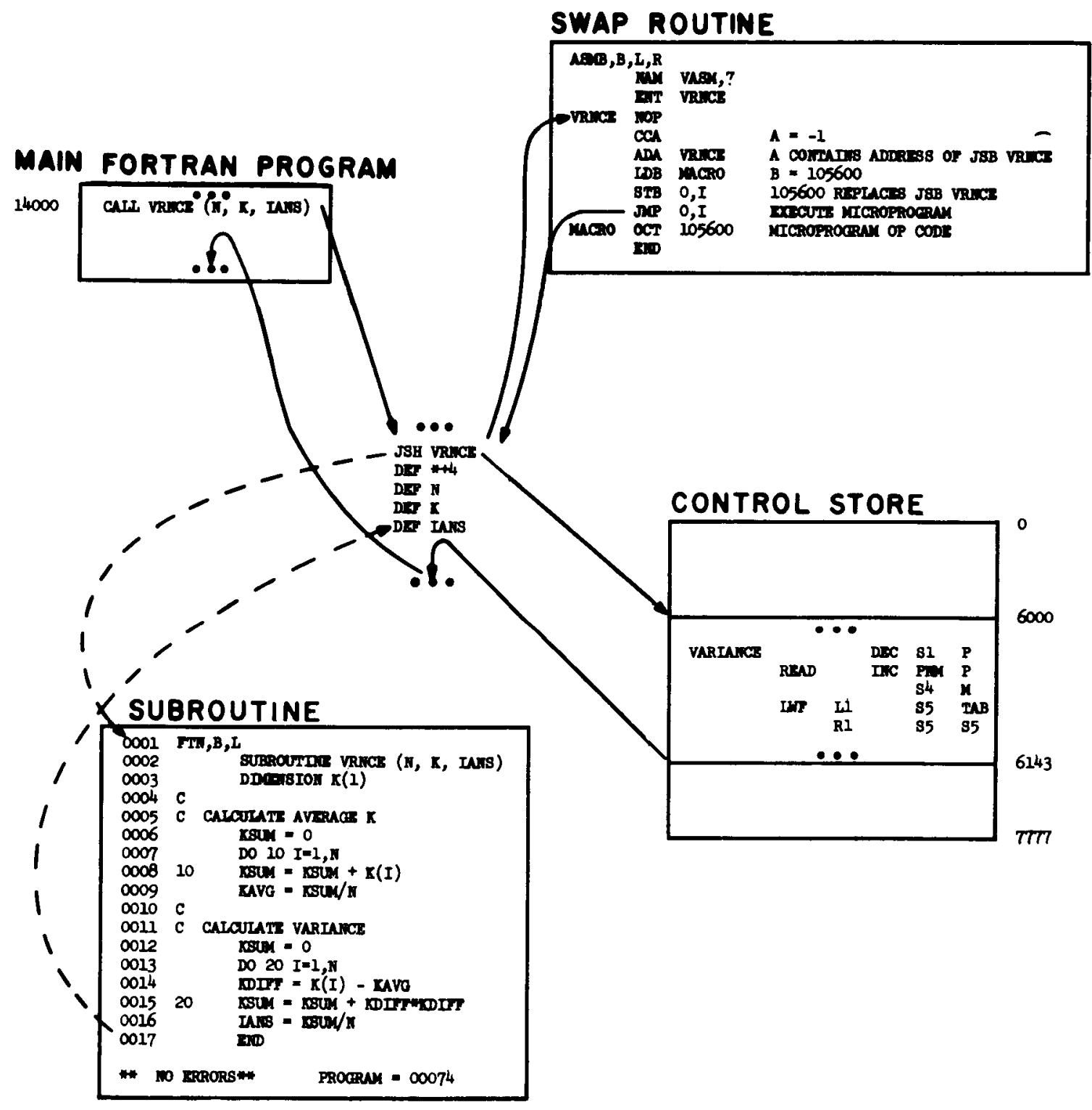

Figure 2. 


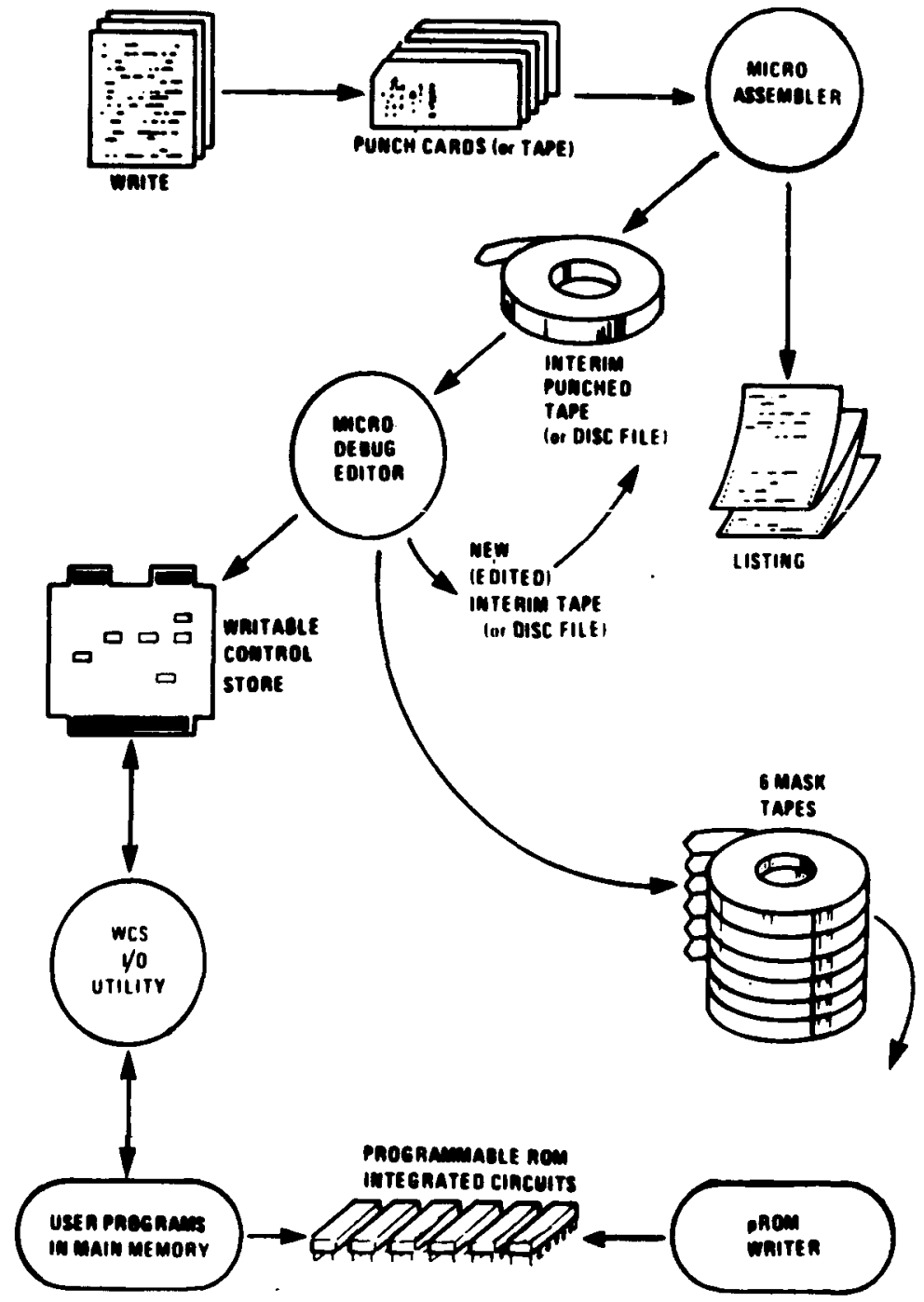

After the microprogram is checked out and operational, one decision remains; should the microprogram be kept in WCS or should it be permanently fixed in a nonmudifiable section of control store, i.e., a PROM (Programmable Read Only Memory)? The advantages of keeping the microprograms in WCS are: (1) WCS may be re-used for many microprograms for many different users. (2) A user may dynamically alter WCS to suit his several application requirements.

The disadvantages of keeping microprograms in WCS are: (1) If power is removed from WCS, the microprograms are lost and must be reloaded. (2) Microprograms in WCS can be destroyed by errant user programs. The advantage of PROM chips is that the microprograms are permanently part of the CPU. The disadvantage of PROM chips is that, if microprograms are updated, new PROM chips must be burned.

PROMs are available for standard applications, such as floating point mathematics, and Fortran code execution.

It shall be noted that selection of what to microcode is extremely important and is not necessarily a trivial task. Three techniques are: (1) Attaching program
Figure 3. analysis devices to the $\mathrm{CPU}-$ most accurate and most expensive. (2) Running program analysis programs on the CPU almost as accurate and considerably cheaper than hardware program analysis devices. (3) Manual periodic halting of the CPU, noting the program counter, and compiling an activity profile-tedious, but minimal material cost. The Hewlett-Packard solution is a program known as ACP (Activity Profile Generator) which programmatically compiles an activity profile representing the percentage of time spent in user specified areas-tobe-analyzed (i.e., blocks of memory). The activity profile is correlated to the programs in these blocks of memory by means of program load maps generated during standard operating system loader procedures. This procedure is shown in Figure 4.

\section{DATA PROCESSING TASKS SUITABLE FOR MICROPROGRAMMING}

Any iterative CPU tasks are prime candidates for microcode. A few examples are: (1) statistical analysisvariance, standard deviation, correlation, . . : (2) trans- 

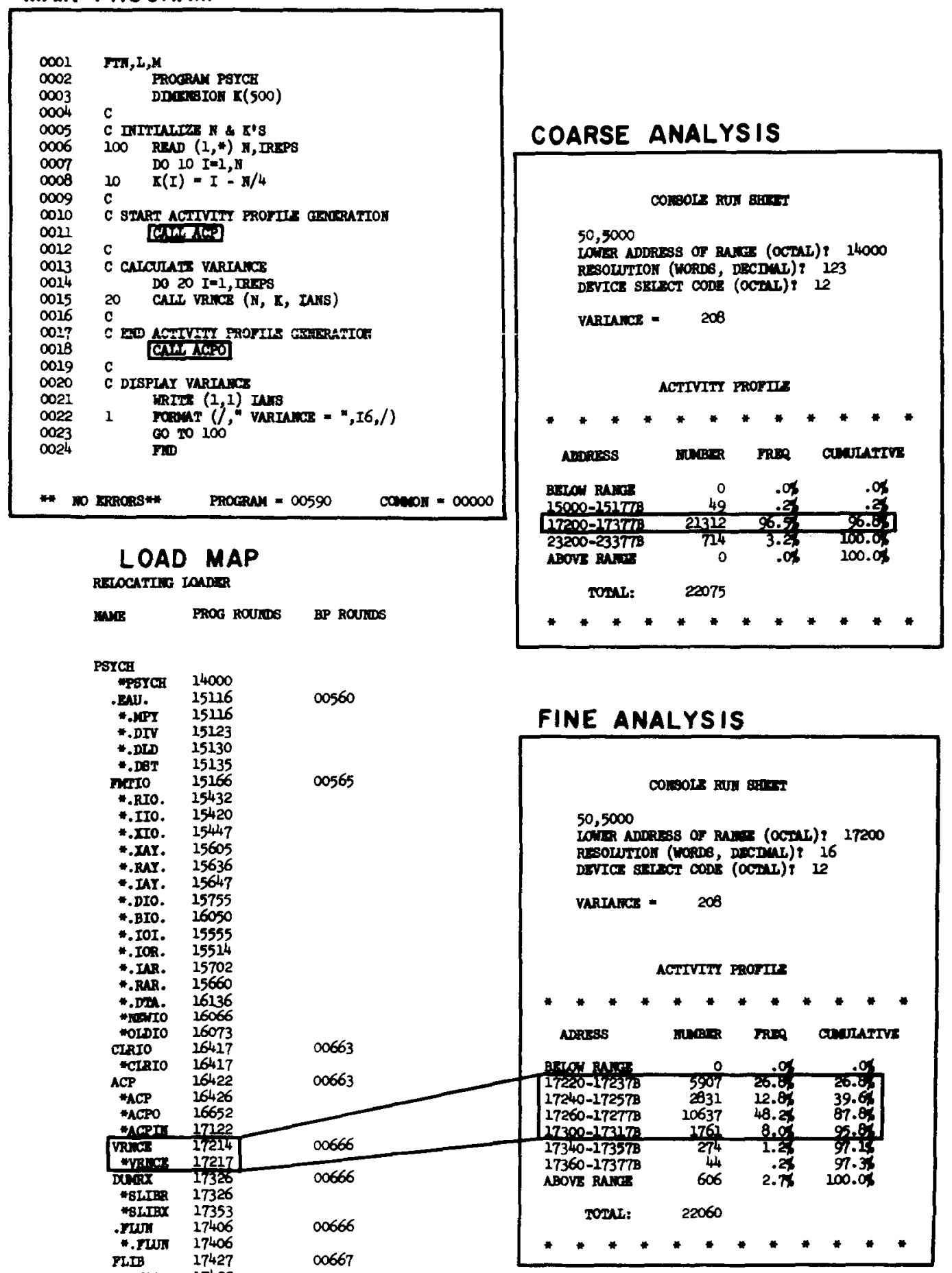

Figure 4.

cendental functions-sine, cosine, tangent, square root, ...; (3) table search-linear, binary, link-list, . . ; (4) sort-bubble, shell, radix-interchange,....: (5) FFT (Fast Fourier Transform). Data gathering functions including (1) pseudo-DMA I/O and (2) privileged $\mathrm{I} / \mathrm{O}$ are also highly suitable for microcode enhancements. Cold start and special loaders should be considered.

Typical improvements realized through microcode en- hancement vary depending on the $\mathrm{CPU}$; for HewlettPackard's 21MX, 5:1 speed reductions are typical.

Statements of CPU tasks in algorithm form (i.e., microcodable form) may be found in many references, for example:

Hart, J. F., et al. Computer approximations. New York: Wiley, 1968.

Knuth, D. E. The art of computer programming (3 
vols.). Reading, Massachusetts: Addison-Wesley, 1968-1973.

The Association for Computing Machinery, Inc. All publications. Address: 1133 Avenue of the Americas, New York, New York 10036.

The Institute of Electrical and Electronics Engineers, Inc. All publications. Address: 345 East 47th Street, New York, New York 10017.

\section{CALCULATION OF "VARIANCE" WITH AND WITHOUT MICROCODE ENHANCEMENT}

Variance, (summation (K-KAVG)**2)/N, is typical of the types of functions that may be profitably microcoded. Using a Fortran subroutine to calculate variance of integer data, run time for 50 data points $(5,000$ repetitions) was $25 \mathrm{sec}$. Substituting microcode for the variance subroutine, the run time was reduced to $5 \mathrm{sec}$. In another calculation, 500 data points were used and the result of the integer calculation performed in the Fortran subroutine was incorrect as the intermediate sums exceeded the integer maximum capacity. However, the microcode routine, being specifically designed to handle double-integer intermediate sums, correctly calculated the variance.

Thus, by using microcode, a customized instruction (variance calculation) achieving reduced execution time ( $25 \mathrm{sec}$ to $5 \mathrm{sec}$ total run time) and special capability (integer calculations requiring intermediate results $>32 \mathrm{~K}$ ) has been developed. Those interested in learning more about microprogramming may attend HewlettPackard's 5-day course, given monthly at Cupertino, California.

\section{REFERENCE}

HLsson. S. S. Microprogramming. Principles and practices. Englewood Clitts. New Jersey: Prentice-Hall. $19^{7} 0$. 